\title{
CAD/CAM transtibial prosthetic sockets from central fabrication facilities: How accurate are they?
}

\author{
Joan E. Sanders, PhD; ${ }^{1-2 *}$ Ellen L. Rogers, $\mathrm{MS}^{1}$; Elizabeth A. Sorenson, MSE; ${ }^{1}$ Gregory S. Lee, PhD; $^{1}$ Daniel C. $^{2}$ \\ Abrahamson, $\mathbf{C P O}^{2}$ \\ Departments of ${ }^{1}$ Bioengineering and ${ }^{2}$ Rehabilitation Medicine, College of Engineering and School of Medicine, Uni- \\ versity of Washington, Seattle, WA
}

\begin{abstract}
This research compares transtibial prosthetic sockets made by central fabrication facilities with their corresponding American Academy of Orthotists and Prosthetists (AAOP) electronic shape files and assesses the central fabrication process. We ordered three different socket shapes from each of 10 manufacturers. Then we digitized the sockets using a very accurate custom mechanical digitizer. Results showed that quality varied considerably among the different manufacturers. Four of the companies consistently made sockets within $+/-1.1 \%$ volume (approximately 1 sock ply) of the AAOP electronic shape file, while six other companies did not. Six of the companies showed consistent undersizing or oversizing in their sockets, which suggests a consistent calibration or manufacturing error. Other companies showed inconsistent sizing or shape distortion, a difficult problem that represents a most challenging limitation for central fabrication facilities.
\end{abstract}

Key words: AAOP, amputee, assessment, digitizer, fabrication, patellar tendon bearing, prosthesis, rehabilitation, residual limb, shape, volume.

\section{INTRODUCTION}

Central fabrication facilities provide custom remote manufacturing services for clinical prosthetists. With central fabrication, a prosthetist uses prosthetic design software (e.g., ShapeMaker ${ }^{\mathrm{TM}}$ [Seattle Limb Systems, Poulsbo, Washington], TracerCAD ${ }^{\mathrm{TM}}$ [Polhemus, Colchester, Vermont], TT Design [Otto Bock, Minneapo- lis, Minnesota], OMEGA Tracer [Ohio Willow Wood, Mt. Sterling, Ohio], BioSculptor [BioSculptor Corporation, Hialeah, Florida]) to design a prosthetic socket for an amputee patient. An undeformed cast or a laser scan of the limb shape is often used as a starting point. Using the software, the prosthetist modifies the original shape into a socket shape and then sends that electronic data file to a central fabrication facility. A facility technician carves a positive model using a computer-controlled lathe and then vacuum-forms onto that shape to create a socket. The socket is then sent back to the prosthetist, typically within 1 to 3 days.

The use of central fabrication has a number of advantages compared with traditional socket fabrication methods. Sockets are made quickly with less technical work required; thus a prosthetist can potentially fit more patients in less time. One clinic reports up to a fourfold

\footnotetext{
Abbreviations: $\mathrm{AAOP}=$ American Academy of Orthotists and Prosthetists, $\mathrm{CAD} / \mathrm{CAM}=$ computer-aided design/computeraided manufacturing, LDT = linear displacement transducer, $\mathrm{NIH}=$ National Institutes of Health, PETG = polyethylene terephthalate glycol, PT = patellar tendon, RVDT = rotary variable differential transducer, $\mathrm{SD}=$ standard deviation.

*Address all correspondence to Joan E. Sanders, PhD, Associate Professor; Bioengineering 355061, Foege N430J, 1705 NE Pacific St, University of Washington, Seattle, WA 98195; 206-221-5872; fax: 206-616-2509.

Email: jsanders@u.washington.edu

DOI: 10.1682/JRRD.2006.06.0069
} 
improvement in efficiency using computer-aided design/ computer-aided manufacturing (CAD/CAM) (central fabrication) over manual (traditional) methods [1]. A duplicate socket can be easily fabricated, and socket shapes can be exchanged between clinicians at different locations. Another advantage is that the prosthetist does not need to buy or maintain the expensive fabrication equipment. In 2001, a higher percentage of prosthetists were using central fabrication than was originally anticipated [2].

However, an issue with central fabrication and a concern to some prosthetists is the quality of the final socket. Is the fabricated socket the same shape as the shape designed on the computer, i.e., the electronic file from which it was created? An inaccurate shape is problematic because it can cause fit problems and necessitate refitting. Further problems may occur later when refitting is necessary, since the documented shape does not reflect the socket shape worn by the patient. For teaching CAD/ CAM to student prosthetists, an accurate shape is essential. Inexperienced student prosthetists have difficulty distinguishing their design errors from central fabrication manufacturing errors.

This research quantitatively compared fabricated socket shapes and CAD electronic data file shapes for a collection of transtibial amputee sockets made by different central fabrication facilities. Our intent was to identify specific errors in socket shape quality and to help identify needed improvements in the central fabrication process or equipment.

\section{METHODS}

\section{Socket Designs}

Sockets designs from three amputee subjects were used in this study. The source of the socket shape files was the ShapeMaker (Seattle Limb Systems, Poulsbo, Washington) prosthetic design software package. According to the software manufacturer, these three examples were actual amputee patient cases created by digitizing undeformed rigid casts of three unilateral amputee residual limbs and then applying the ShapeMaker transtibial (below-knee) rectification templates to them. Each of the three files was made up of 72 points per slice, with between 48 and 68 slices per socket (socket $A=68$, socket $B=50$, and socket $C=48$ ). Socket lengths from the mid-patellar tendon (PT) to the distal end of the socket were socket $\mathrm{A}=12.8 \mathrm{~cm}$, socket $\mathrm{B}=14.2 \mathrm{~cm}$, and socket $C=15.7 \mathrm{~cm}$. Socket shapes are shown in Figure 1(a)-(c). The socket shapes were stored in American Academy of Orthotists and Prosthetists (AAOP) format. This file format consists of header information on slice parameters and then a series of coordinates for each slice. An equal number of points are in each slice.

\section{Central Fabrication Facilities}

Each of 10 selected central fabrication facilities was contracted to manufacture test sockets for each of the three socket designs. Thus, a total of 30 sockets were created. All facilities could manufacture sockets from AAOP-formatted electronic shape files sent to them by email. The manufacturers were instructed not to trim the test socket but to cut straight across at the proximal end, since this simplified the digitization process. The companies selected were from eight different U.S. states. They were selected randomly from lists on the Internet. The facilities ranged in production size from approximately 130 sockets a month to 190 sockets a month, according to company representatives. Socket prices ranged from $\$ 98$ to $\$ 165$ in 2006 dollars, not including tax or shipping. Sockets arrived between 2 and 11 days from when the electronic shape files were sent. All sockets were made of polyethylene terephthalate glycol (PETG) except sockets from company 5 , which were made of copolyester. One socket was ordered at a time, as opposed to ordering all three sockets together. All sockets were blister-formed except those from company 9, which were draped. All manufacturers used foam-positive models except for company 7 , which used a plaster and cornstarch combination. One manufacturer (company 1) reduced the computer model by 1 ply to compensate for a 1-ply sock used during vacuum-forming, and another company (company 9) reduced the model globally (by an unspecified amount). No other manufacturer performed compensations. The sock ply or coating used and the degree of hand sanding used to smooth the positive model varied by manufacturer (Table 1).

\section{Socket Digitization}

The sockets were digitized with a custom mechanical digitizer described in detail elsewhere [3] (Figure 2). The digitizer was a spring-loaded arm connected to a rotary variable differential transducer (RVDT) and a servocontrolled linear slide rail on a base frame. The springloaded arm was made up of a sapphire ball that contacted 


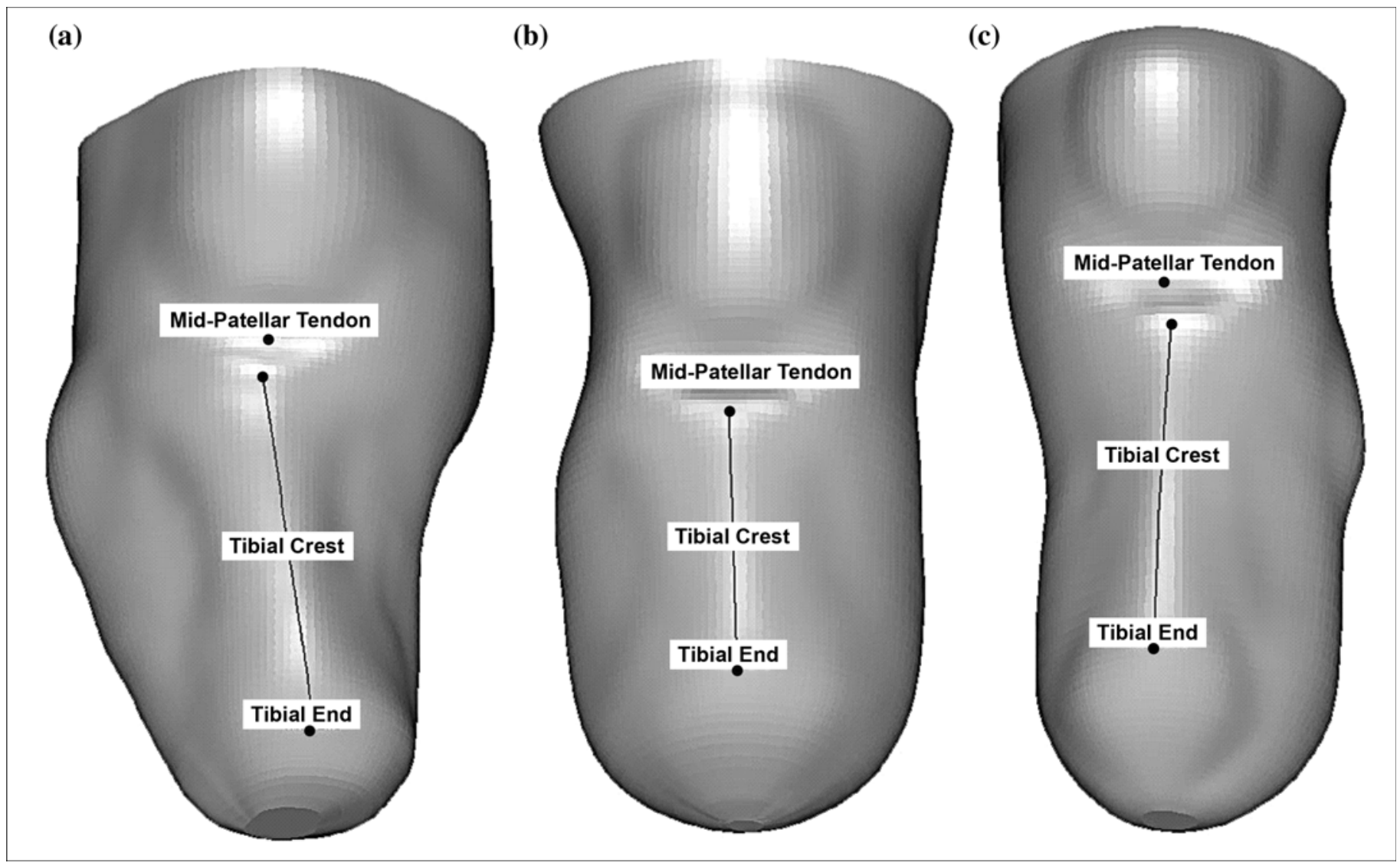

Figure 1.

Socket shapes used for evaluation: (a) socket A, (b) socket B, (c) socket C.

Table 1.

Company manufacturing practices.

\begin{tabular}{cll}
\hline Company & \multicolumn{1}{c}{ Sock Ply Used } & \multicolumn{1}{c}{ Hand Sanding? } \\
\hline 1 & 1-ply sock & Yes, to smooth carve lines if necessary \\
2 & Nylon + soak model in water & No \\
3 & Nylon & Yes, everywhere, to remove loose foam from model \\
4 & Nylon (very thin panty hose) & $\begin{array}{l}\text { New carver leaves surface fairly smooth; minor touch up } \\
\text { only if necessary to remove carve lines }\end{array}$ \\
5 & Sock + silicone to prevent plastic from sticking & No \\
6 & None, but air holes drilled and foam covered with & Yes, light hand sanding to smooth carve lines \\
7 & silicone/liquid to prevent plastic from sticking & \\
8 & None & Yes, to smooth carve lines \\
9 & Nylon & Yes, very light if needed; typically not needed \\
10 & Nylon & Yes, to remove carve lines; no modification \\
\hline \hline
\end{tabular}




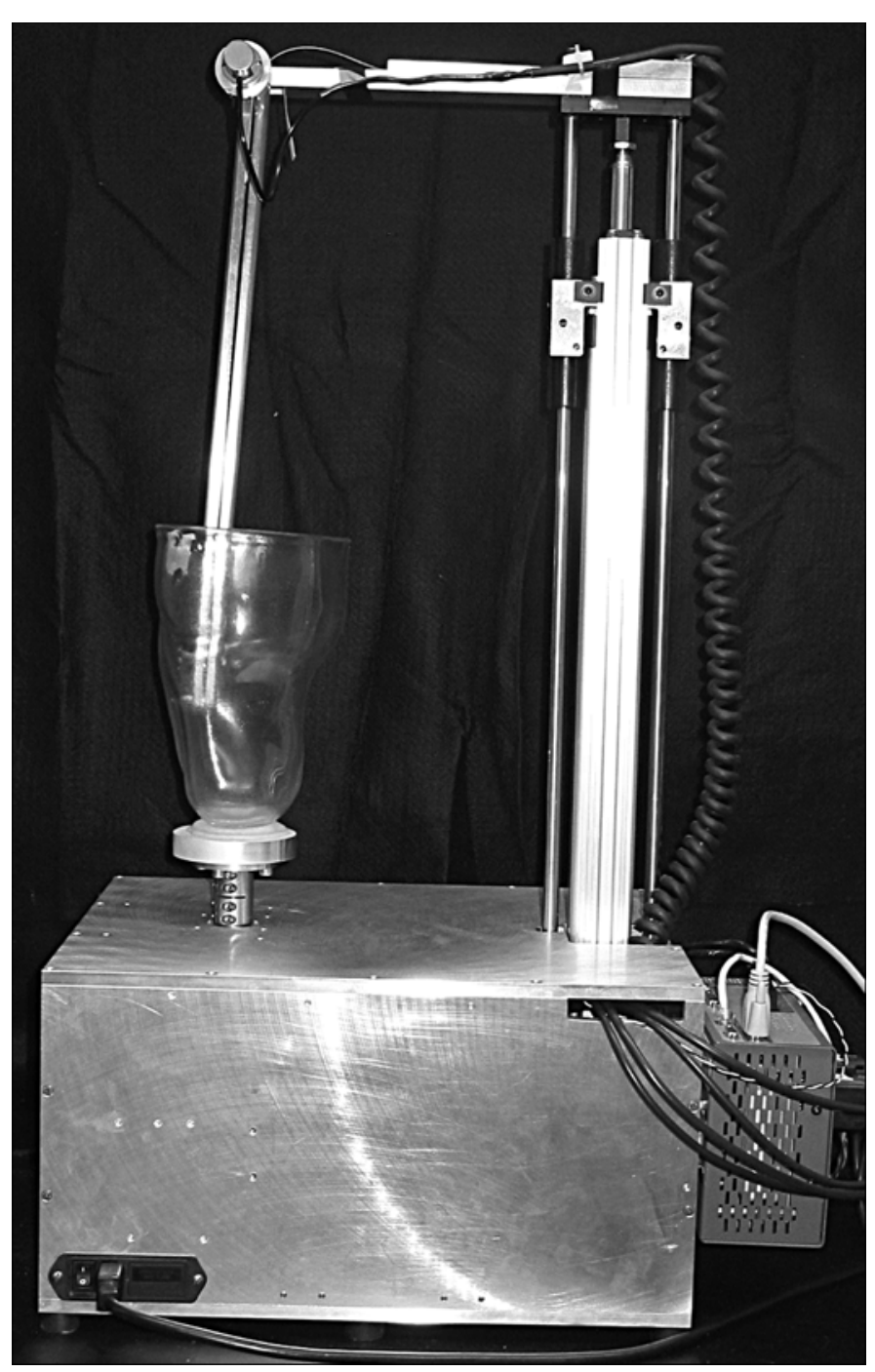

Figure 2.

Custom mechanical digitizer used for socket shape assessment.

the socket, Vee Jewel pivots, and a four-bar linkage. The stylus was modified slightly from that described previously [3], in that the piece supporting the sapphire ball was reduced in size and the sapphire ball was glued to the modified support piece instead of being clamped in place. These changes ensured sockets with high curvature regions were accurately digitized. The slide rail had a linear displacement transducer (LDT) for position measurement. The socket was rotated with a servomotor, which was positioned in the base of the digitizer and equipped with an angular encoder for position measurement. The position of the sapphire ball was precisely measured with data from the RVDT, LDT, and angular encoder.
In a scan, 800 points per slice were recorded at a slice spacing of $1.75 \mathrm{~mm}$. These sampling intervals produced sufficient resolution for the needs of this project and were not excessively time-consuming to run. Approximately 3 hours were required to scan one socket.

In this research, the digitizer recorded the position of the stylus ball as it contacted the socket at equal angular intervals in each slice. Angular positions were identical for all slices; thus all points for each angular position were in the same $r$-z plane. The position of the center of the stylus ball was recorded, as opposed to its edge, since the center position measurement in the $r$-z plane was not affected by where on the ball the socket made contact. The center position was always a radius away from the socket surface at an angle perpendicular to the socket surface in the $r$-z plane. All points in the same $r$-z plane were connected with Cartesian B-splines. Using point projection, we calculated a B-spline at a perpendicular distance to the stylus ball radius away from that spline, representing the shape of the measured socket surface. Once all the B-splines were established, Cartesian B-spline tubular surfaces were then fitted to the data. The use of Bsplines simplified comparison between electronic AAOP file data and digitizer data, since no a priori one-to-one correspondence between points on the two surfaces was required.

We calibrated the custom digitizer using a precisely machined axisymmetric calibration object with six distinct regions of constant internal diameter. The diameters were $25,35,45,55,65$, and $75 \mathrm{~mm}$. The RVDT on the arm yielded a linear relationship between voltage and arm angle. The known radii were used for calculating the coefficients of the linear relationship between voltage and arm angle. The linear relationship was then used for converting the voltage data into radii (in millimeters).

We conducted bench tests using an axisymmetric test object with varying radii. The object had regions with slopes relative to horizontal of $25^{\circ}, 35^{\circ}, 45^{\circ}, 55^{\circ}, 65^{\circ}$, $75^{\circ}$, and $85^{\circ}$. The minimum of $25^{\circ}$ was approximately $10^{\circ}$ less than the shallowest slope region of any of the three socket designs tested. After digitization, the measured slopes of the axisymmetric test object were compared with the known slopes.

To prepare for digitizing sockets, we affixed the sockets to $16.5 \mathrm{~mm}$-thick aluminum plates using Orthocryl sealing resin and hardening powder (Otto Bock). This material is typically used in prosthetics to bond test sockets to support blocks. The sockets were affixed such that 
the socket central axis was visually aligned with the center of the mounting plate. The plates were equipped with a $50.8 \mathrm{~mm}$ (2 in.) bolt circle for direct attachment to a stand with a central axle that fit into a single-slit collar on the digitizer base (Figure 2).

The beginning point of scanning, which was at the bottom of the socket, was selected to be as low as the stylus could go without scraping the bottom of the socket. The end of the scan was the highest point on the socket where the stylus could fully rotate without losing contact with the surface. Only the region of the socket comparable with the electronic AAOP shape file, which ended approximately $1 \mathrm{~cm}$ above the knee, was used for evaluation.

We conducted evaluation tests to assess repeatability and the effects of socket positioning on performance. Repeatability was assessed by digitizing the same socket three times without removing it from the digitizer. To assess the influence of socket position, we conducted eight test scans and one reference scan of the socket. For the reference scan, the socket was affixed to the support block such that the socket axis was visually aligned with the center of the mounting plate. We conducted the test scans angled $2.5^{\circ}$ in the anterior, posterior, lateral, and medial directions by tilting the support plate relative to the digitizer base. The $2.5^{\circ}$ resulted in approximately 1.0 $\mathrm{cm}$ of axis misalignment at the proximal end of the socket. Test scans with $0.5 \mathrm{~cm}$ of exclusively translational misalignment in the anterior, posterior, medial, and lateral directions were also conducted. These tests required that the socket be removed and then reattached to the mounting plate. Shape differences between each of the nine scans and the electronic shape file were assessed.

\section{Shape Analysis}

The digitized socket shape was aligned with the electronic AAOP file shape with algorithms described in detail elsewhere [4]. In short, the shape alignment algorithm was an optimization procedure that used a weighted linear combination of maximized global similarity and maximized local shape similarity. To maximize global similarity, we used the minimum weight-matching variant of the bottleneck-matching method. This matching method minimized the sum of the absolute differences between the two corresponding radii of the two point sets (i.e., the mean absolute difference) [5]. Clinically, this process corresponds to minimizing the volume difference between the two shapes. Then we ran an algorithm to maximize local shape similarity by summing the dot products of corresponding surface normals. The weighted linear combination of the global and local shape similarity measures was shown to produce rootmean-square errors of $0.04 \mathrm{~mm}$ in translation and $0.06^{\circ}$ in rotation for four residual-limb shape-change conditions tested [4].

Comparisons were made between the socket shapes and electronic AAOP files for volume, cross-sectional area, radii, and PT diameter. Cross-sectional area was calculated for each slice with linear interpolation between adjacent points in a slice (72 points per slice, consistent with the electronic AAOP files). Volume was calculated as the sum of the cross-sectional areas multiplied by the slice spacing. The location of the PT was identified by visual inspection of the electronic AAOP file. We used the most anterior and most posterior points on that slice to compare PT diameter between the socket shape and electronic AAOP file shape.

\section{RESULTS}

\section{Digitization Error}

Results from the digitizer evaluation showed that calibration parameters were consistent over the course of a day within the resolution limits of the system. The digitizer was calibrated each morning before use.

Test results using the axisymmetric test object showed slightly reduced performance at very shallow angles (relative to horizontal) than at steep angles. Measured slopes were within $\pm 0.5^{\circ}$, the accuracy of the machined test object, for the angle range of $45^{\circ}$ to $85^{\circ}$ relative to horizontal. For $35^{\circ}$, the error was $0.8^{\circ}$; for $25^{\circ}$, the error was $0.9^{\circ}$.

Repeatability tests conducting with a representative test socket helped to establish the performance of the system. Standard deviations (SDs) in the results for the seven features of interest here were $\Delta$ volume $=0.06$ percent, percentage of points within $1.00 \mathrm{~mm}$ of AAOP design $=0.2$ percent, radial minimum error $=0.08 \mathrm{~mm}$, radial maximum error $=0.01 \mathrm{~mm}$, radial mean error $=$ $0.01 \mathrm{~mm}$, radial SD of mean error $=0.00 \mathrm{~mm}$, and PT diameter difference $=0.08 \mathrm{~mm}$.

The digitizer performed well under misalignment conditions, which showed results comparable with those from the repeatability tests. Results for the reference and misalignment tests are shown in Table 2. SDs from the nine scans for the seven features of interest were $\Delta$ volume $=$ 
JRRD, Volume 44, Number 3, 2007

Table 2.

Socket misalignment test results.

\begin{tabular}{|c|c|c|c|c|c|c|c|}
\hline \multirow[b]{2}{*}{ Orientation } & \multirow{2}{*}{$\begin{array}{c}\Delta \text { Volume } \\
\text { Socket-AAOP } \\
\text { AAOP } \\
(\%)\end{array}$} & \multicolumn{5}{|c|}{ Radius } & \multirow[b]{2}{*}{$\begin{array}{l}\text { PT Diameter } \\
\text { Socket-AAOP } \\
(\mathbf{m m})\end{array}$} \\
\hline & & $\begin{array}{c}\text { \% of Points Within } \\
\pm 1.00 \mathrm{~mm} \text { of } \\
\text { AAOP Design }\end{array}$ & $\begin{array}{l}\text { Min } \\
\text { Error } \\
(\mathbf{m m})\end{array}$ & $\begin{array}{l}\text { Max } \\
\text { Error } \\
(\mathbf{m m})\end{array}$ & $\begin{array}{l}\text { Mean } \\
\text { Error } \\
(\mathrm{mm})\end{array}$ & $\begin{array}{l}\text { SD of Mean } \\
\text { Error } \\
(\mathrm{mm})\end{array}$ & \\
\hline Reference & 0.03 & 91.2 & -3.36 & 1.80 & 0.04 & 0.60 & -0.26 \\
\hline Ang Anterior & 0.11 & 91.6 & -3.22 & 1.95 & 0.06 & 0.59 & -0.30 \\
\hline Ang Posterior & -0.05 & 89.8 & -3.25 & 2.07 & 0.02 & 0.62 & -0.16 \\
\hline Ang Lateral & 0.01 & 90.5 & -3.12 & 1.89 & 0.03 & 0.61 & -0.12 \\
\hline Trans Anterior & 0.02 & 91.0 & -3.15 & 1.74 & 0.04 & 0.61 & -0.23 \\
\hline Trans Posterior & 0.07 & 91.2 & -3.28 & 1.79 & 0.05 & 0.60 & -0.30 \\
\hline Trans Medial & 0.06 & 90.7 & -3.21 & 1.86 & 0.05 & 0.61 & -0.17 \\
\hline Trans Lateral & -0.03 & 90.4 & -3.26 & 1.76 & 0.03 & 0.61 & -0.27 \\
\hline
\end{tabular}

AAOP = American Academy of Orthotists and Prosthetists, Ang = angular misalignment direction, Max = maximum, Min = minimum, PT = patellar tendon, SD $=$ standard deviation, Trans = translational misalignment direction.

0.05 percent, percentage of points within $1.00 \mathrm{~mm}$ of AAOP design $=0.6$ percent, radial minimum error $=0.07$ $\mathrm{mm}$, radial maximum error $=0.11 \mathrm{~mm}$, radial mean error $=$ $0.01 \mathrm{~mm}$, radial SD of mean error $=0.01 \mathrm{~mm}$, and PT diameter difference $=0.06 \mathrm{~mm}$. Based on these tests, the resolutions for $\Delta$ volume and PT diameter difference were reduced for the results presented in the next sections.

\section{Volume Difference}

Analysis of volume differences between the sockets and the electronic AAOP files showed that 20 of the 30 sockets had volumes that were within a range of -1.1 to +1.1 percent of the electronic AAOP shape files (Figure 3; Table 3, column 3). The basis for using a 1.1 percent criterion is that with a $150 \mathrm{~mm}$ length/50 mm radial residual-limb model [6], a 1.1 percent volume change corresponds to approximately 1 sock ply. The other 10 sockets were within windows of -2.8 to -1.2 percent and +1.5 to +5.8 percent. Eighteen sockets had negative volume difference values, which means they were smaller than the electronic AAOP files. Only 5 of those 18 had volume differences greater than -1.1 percent.

\section{Company Dependence}

For four of the companies (companies 1-3 and 5), all three sockets were within a range of -1.1 to +1.1 percent volume difference of the electronic AAOP files. For one company (company 10), all three sockets were outside of the -1.1 to +1.1 percent range. For the other five compa-

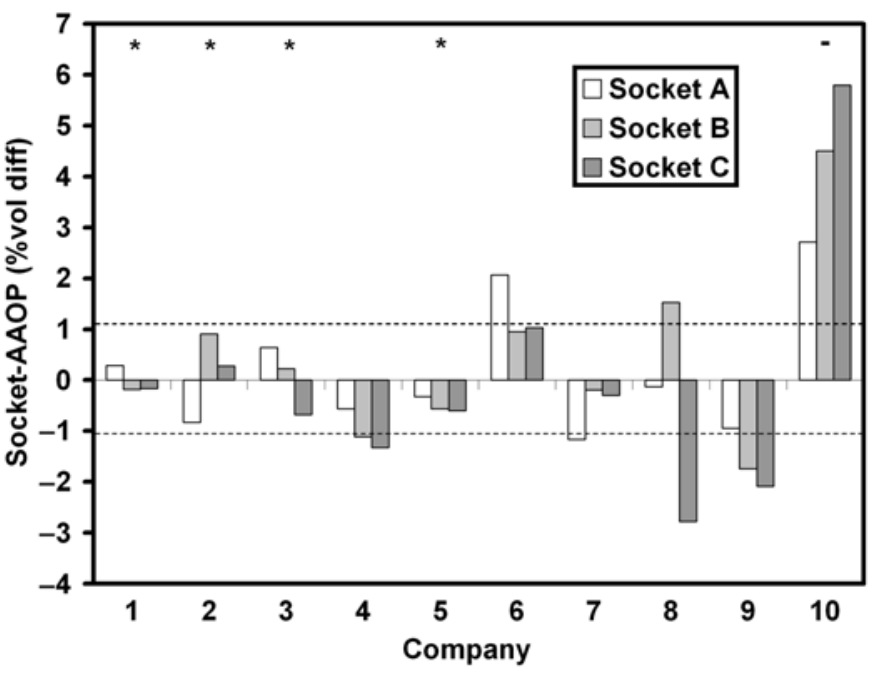

Figure 3.

Percentage volume differences between sockets and electronic American Academy of Orthotists and Prosthetists (AAOP) files. Dashed lines at $1.1 \%$ indicate an approximately 1 -ply difference. $*=$ Companies within $-1.1 \%$ to $+1.1 \%$ error for all sockets. - = Company outside $-1.1 \%$ to $+1.1 \%$ error for all sockets.

nies (companies 4 and 6-9), one or two of the three sockets were within the -1.1 to +1.1 percent range and the others were not. One company (company 8) had one socket with a volume greater than +1.1 percent of the electronic AAOP file and another socket with a volume less than -1.1 percent of the electronic AAOP file.

Three of the four companies that were within the -1.1 to +1.1 percent volume difference range for all sockets 
Table 3.

Performance results for all sockets.

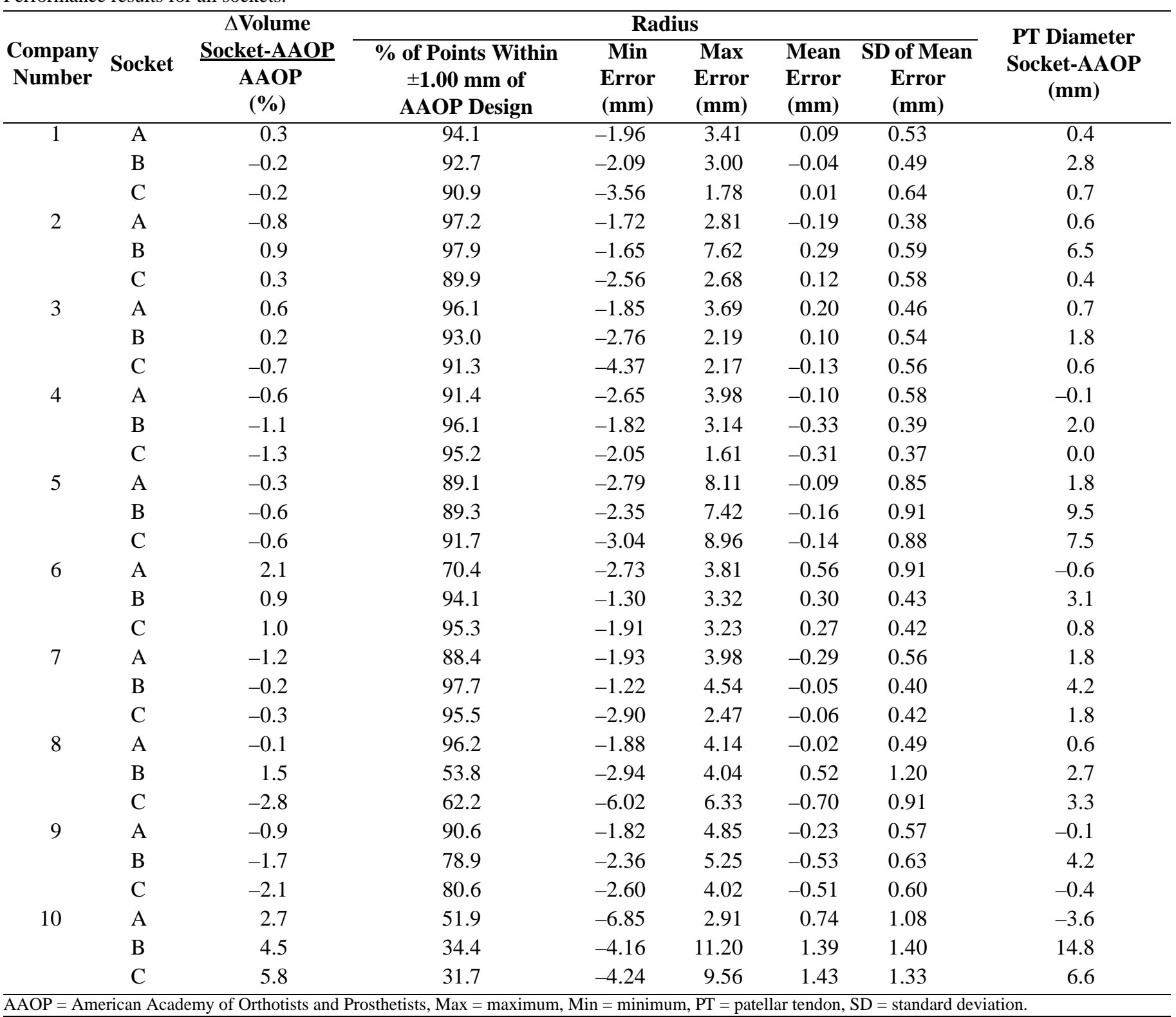

(companies 1, 3, and 5) also had mean radial errors within $\pm 0.25 \mathrm{~mm}$ of that of the designed socket (Table 3, column 7). Fernie and Holliday reported that the thickness of a 5-ply sock was $1.25 \mathrm{~mm}$ [6]. Thus, in this article, we assume that 1 sock ply corresponds to $0.25 \mathrm{~mm}$. Not all sock plies take up the same amount of volume; thus this thickness is an arbitrary specification. Of these three companies, two of them (companies 1 and 3) had over 90 percent of the socket surface within $\pm 1.00 \mathrm{~mm}$ radial distance of the designed surface. Company 5 had relatively large radial SDs of the mean and maximum radial errors. This result indicates that the error range was relatively wide for company 5 .

\section{Error Consistency Among Sockets from Same Company}

For some companies, the direction of the error was consistent among the three sockets. Two companies showed consistently larger sockets (companies 6 and 10), while four companies showed consistently smaller sockets (companies 4, 5, 7, and 9). A consistent direction of 
the error coupled with a relatively low SD of the mean radial error (Table 3, column 8), as occurred for company 4 , suggests an overall offset in the shape for these sockets. In other words, the sockets were consistently smaller or consistently larger throughout the surface than the electronic AAOP files.

\section{Error Consistency Within a Socket}

One company (company 5) had good volume fit results for all sockets (socket volumes within $-1.1 \%$ to $+1.1 \%$ of the electronic AAOP files), but a large range in radii differences and a large SD of the mean radial error (Table 3, columns 5-6 and column 8, respectively). This result suggests that some socket regions were much smaller and some were much larger than the electronic AAOP file.

\section{Cross-Sectional Area Error}

Looking at local shape differences more critically by comparing cross-sectional areas down the lengths of the sockets shows the differences. For socket A, company 3 achieved a very good fit (Figure 4(a)), while the socket of company 6 was too large over the entire length except at the distal end (Figure 4(b)). The socket of company 5 was too small distally but too large proximally beneath the PT (Figure 4(c)).

\section{Local Shape Differences}

Shape differences occurred locally, with the largest errors typically at the PT (Table 3, column 9). One socket (company 10, socket B) showed $>14 \mathrm{~mm}$ of oversizing at this location. The popliteal fossa region, another high curvature area, also showed error (Figure 5(b) and (d)). Sockets were smaller than the electronic AAOP files over the crest of the tibia for almost all sockets (except those for Companies 6 and 10), though the degree of error varied considerably by manufacturer. For example, socket $\mathrm{C}$ from company 9 (Figure 5(a)) showed up to $3 \mathrm{~mm}$ of error over the crest of the tibia while socket $\mathrm{C}$ from company 2 (Figure 5(c)) showed $<1 \mathrm{~mm}$ of error over the tibial crest.

\section{DISCUSSION}

We compared centrally fabricated socket shapes and electronic AAOP shape files to assess the accuracy of the central fabrication process for 10 selected manufacturers. This effort is relevant to the prosthetics industry because (a)

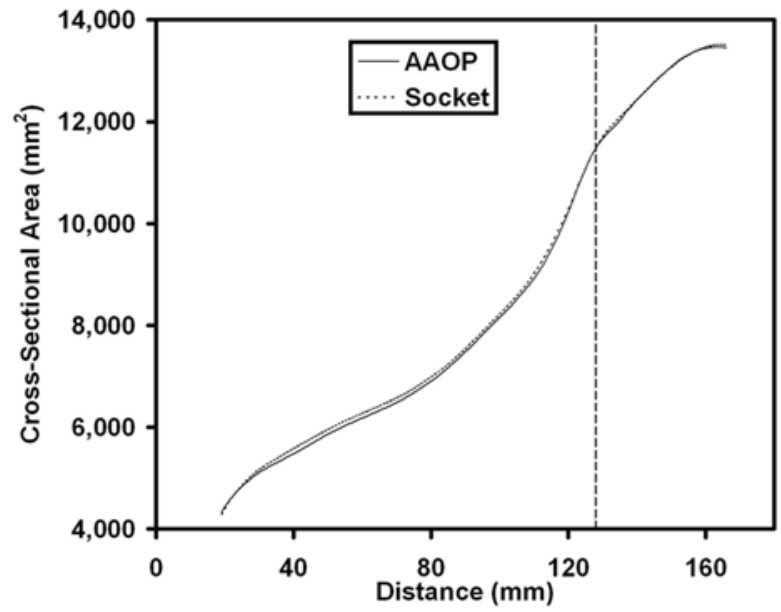

(b)

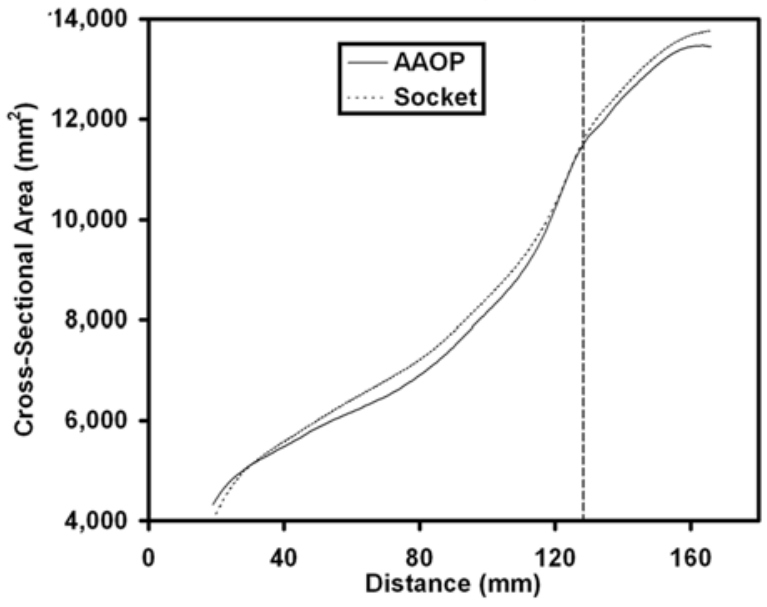

(c)

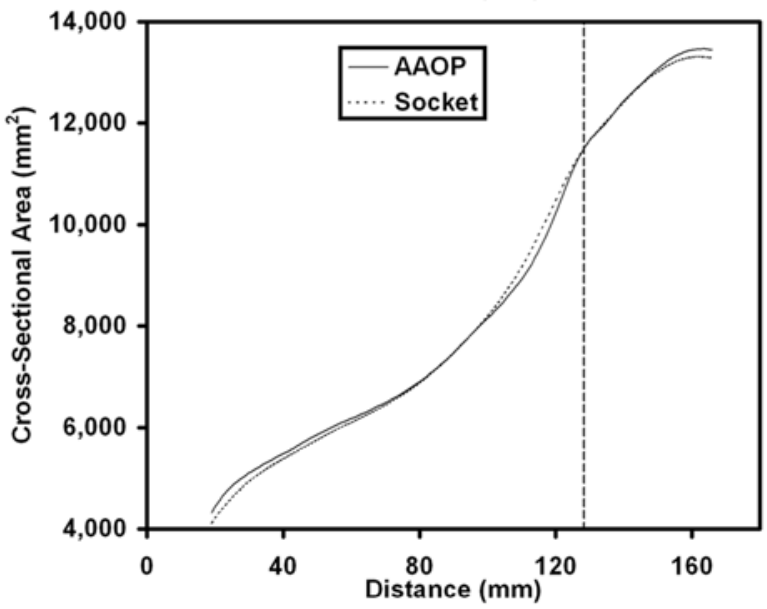

Figure 4.

Cross-sectional area vs distance plots for socket A illustrating different kinds of error. Solid line = electronic American Academy of Orthotists and Prosthetists (AAOP) file. Dashed line = socket. (a) Socket matches electronic AAOP file (company 3) well. (b) Socket is too large over entire socket length except at very distal end (company 6). (c) Socket is too small distally but too large further proximally beneath patellar tendon (company 5). Dashed vertical line identifies patellar tendon location. 
(a)

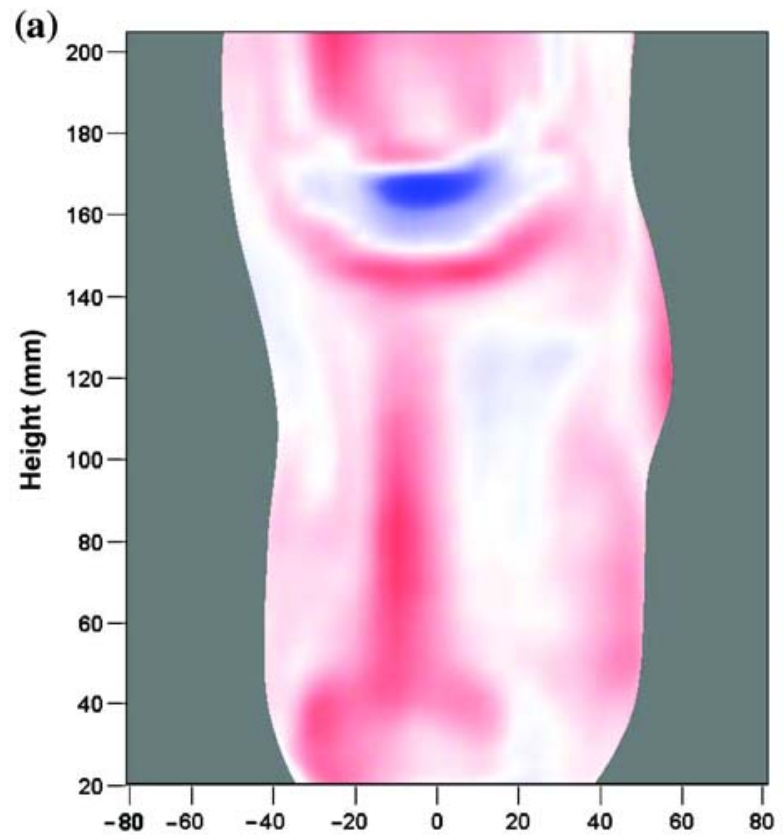

(c)

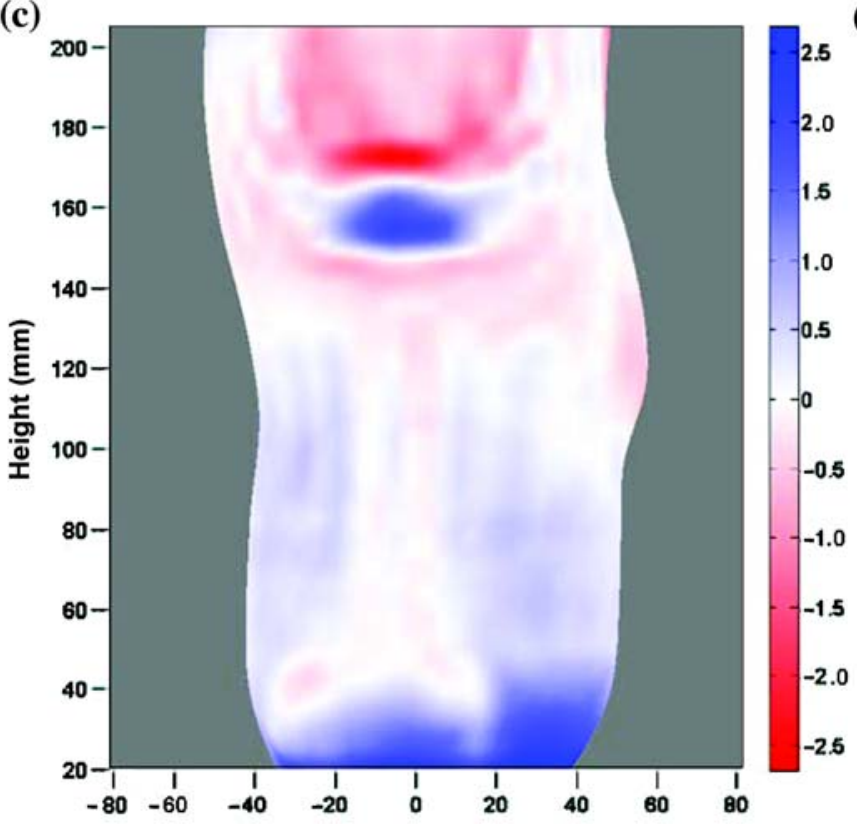

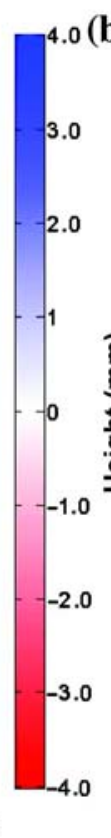

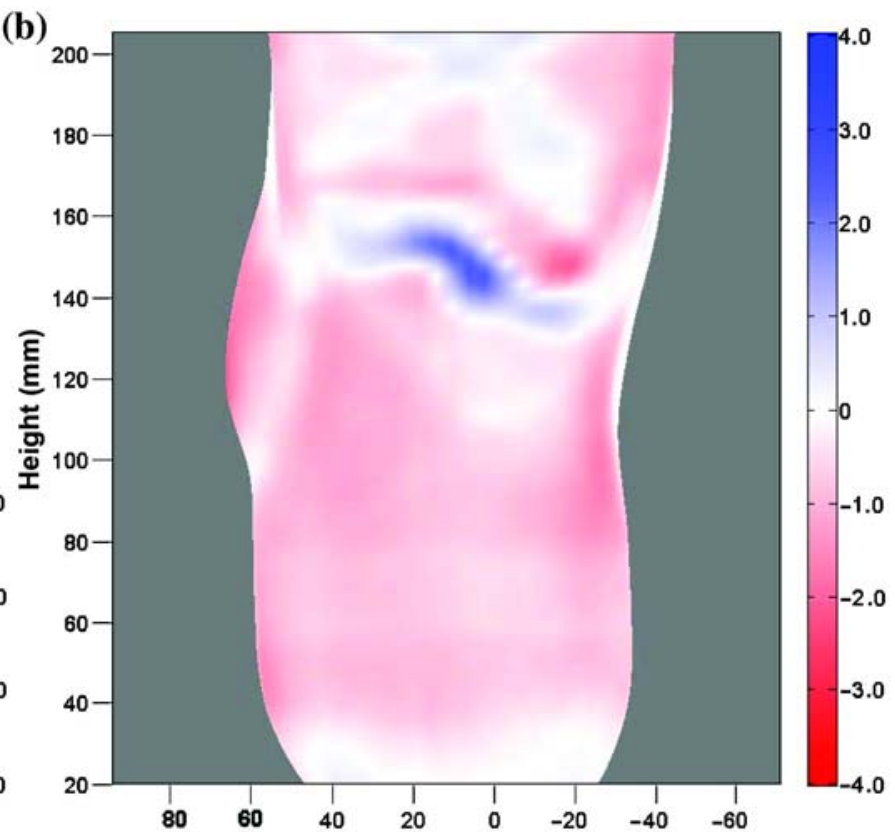

(d)

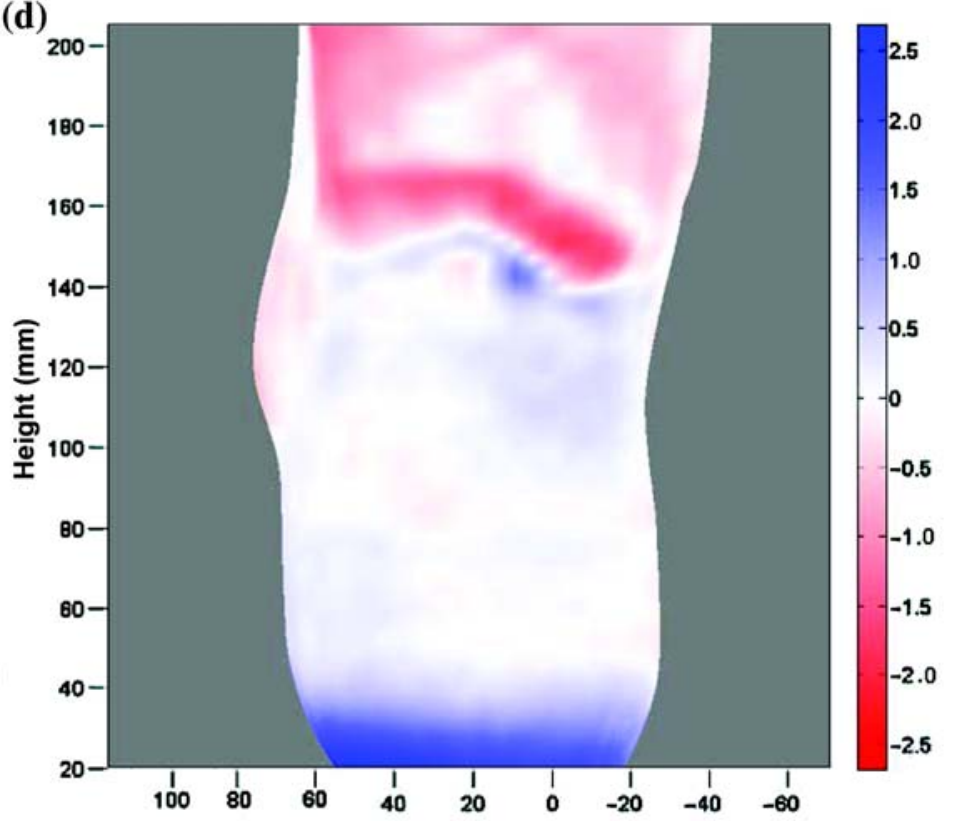

Figure 5.

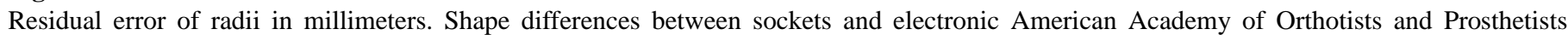

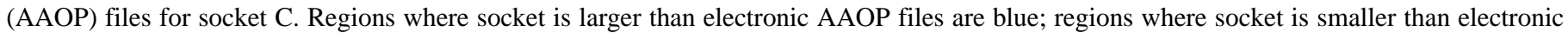
AAOP file are red. (a)-(b) Company 9 showed up to $3 \mathrm{~mm}$ error over crest of tibia. (c)-(d) Company 2 showed <1 mm error over tibial crest.

the results provide insight into the limitations in central fabrication and thus identify areas of needed improvement.

Though evaluation tests showed that the digitizer/shape alignment system performed very well, bench test results showed that, compared with other areas, very high socket curvature regions in the $r$-z planes were not measured as accurately. Axisymmetric test object results showed greater measurement errors for the $25^{\circ}$ and $35^{\circ}$ sections compared with the $45^{\circ}, 55^{\circ}, 65^{\circ}, 75^{\circ}$, or $85^{\circ}$ regions. Furthermore, results from the socket angular misalignment tests (Table 2) showed greater differences for the angular anterior versus angular posterior scans compared with any other 
test pair. Thus, a greater measurement error was likely in the anterior portion of slices near the PT than in other regions. The greater variability in misaligned scan tests (Table 2) for PT diameter difference than for the other calculated features further supports this interpretation. The slice used to calculate PT diameter difference was likely one of the most affected. Changes in other features, for example socket volume, were minimally influenced by this error because the region affected was too small. Though this error is present, it is not large enough to substantially affect the data or the interpretation discussed in the following paragraphs.

Another source of error related to socket misalignment stems from an off-center reference axis. When the socket was positioned in the digitizer such that the $z$-axis passed far from the centroids of the slices, as in the misalignment test scans, the stylus ball did not contact the socket along the $r$-axis. Unlike in the $r$-z plane in which this stylus contact issue was corrected (see "Methods" section), no correction was made in the transverse plane-an error resulted. Because we were able to repeatedly position the sockets well within the misalignment ranges used for testing and because those test results showed relatively little error, we considered this correction unnecessary. Thus based on these evaluation test results, these data may be used to identify similarities and differences in performance among the different central fabrication facilities tested.

The data show that quality varied considerably among the different manufacturers. Two of the manufacturers (companies 1 and 3) had consistently good socket volume, radii, and shape matches. The volume errors of $<1.1$ percent and mean radial errors of $<0.25 \mathrm{~mm}$ for all sockets of companies 1 and 3 corresponded to $<1$-ply sock difference, assuming 1 sock ply is $0.25 \mathrm{~mm}$, between the fabricated sockets and the electronic AAOP files. One other company (company 10) had consistently poor matches. The volume error of 5.8 percent and mean radial error of $1.43 \mathrm{~mm}$ for company 10 , socket C, corresponded to almost a 6-ply oversizing, which approaches clinical recommendation for a new socket. The other seven companies showed inconsistent performance. Thus the data presented here do not show a consistent problem with all of central fabrication. Instead, they indicate that some companies consistently make good sockets, some companies consistently make poor sockets, and some companies sometimes make good sockets. Prosthetists need to choose wisely when selecting a central fabrication facility.

The inconsistent performance among manufacturers complicates the acceptance of central fabrication and CAD/CAM into clinical practice. Depending on which manufacturer is selected and when the socket is ordered, a prosthetist may receive a good, mediocre, or poor socket. Furthermore, the inconsistent performance adds confusion to the teaching and learning of CAD/CAM. Students have difficulty distinguishing their own design errors from central fabrication manufacturing errors.

Careful inspection of the data helps identify the different kinds or errors that occurred. Company 4 consistently made sockets that were too small, ranging from 0.6 to 1.3 percent volume undersized. The consistent direction of the mean radial error and the relatively low SDs suggest a consistent radial underestimate over the entire socket, averaging 0.10 to $0.33 \mathrm{~mm}$. This result could reflect the type of wick sock used coupled with the lack of compensation. However, before this conclusion can be definitively made, a systematic analysis varying only the sock type or only the presence of compensation would need to be conducted. While the consistent undersizing is problematic, accommodating for it in clinical practice is relatively easy by simply enlarging the overall socket size in the electronic AAOP file before sending it to this manufacturer. The relevance to the manufacturer is that a calibration error in their fabrication process is likely, either in carving or vacuum-forming, and needs to be corrected to overcome this limitation.

A more difficult problem to accommodate is that of company 8 . One time, this manufacturer's socket was more than 1 ply oversized, and another time, the socket was more than 1 ply undersized. Accounting for this inconsistency is almost impossible in clinical practice and represents the worst kind of error.

Another difficult problem to accommodate in clinical fitting is that of socket-shape distortion. Sockets from company 5, for example, were consistently too small in cross section distally. Clinically, this error would increase interface loading at this location, typically a sensitive area. This result might be a reflection of the copolyester used by company 5 (as opposed to PETG). However, a systematic analysis in which only the polymer was varied while all other variables remained constant would need to be conducted for this conclusion to be determined.

A consistent result among manufacturers was a relatively high error at the PT. The PT area was oversized for 
25 of the 30 sockets. This result reflects the challenges in first carving and then vacuum-forming over such highcurvature regions. Even the best manufacturers (companies 1 and 3) still had some distortion in this area.

While inspection of the data just as described provides insight into the sources of errors, the reason so much variability exists among different manufacturers remains an open question. Possibly, the results may reflect either the quality and/or maintenance schedule of the carvers, or the features listed in Table 1 or described in the "Methods" section may influence the results. While information about different company manufacturing practices is interesting here, the study design does not allow us to quantify the relative influence of each design variable. A logical next step would be to evaluate each variable (e.g., different socket material, different positive material, forming method [blister vs drape], sock ply, sock type, sock compensation, sanding) individually in a systematic statistical sense. All other variables but the test variable would need to be kept constant. Also logical would be to evaluate positive models made from different carvers by using methods similar to those described in this article. This effort would help distinguish if the error was from carving or vacuum-forming. With the sources of error pinpointed, recommendations can be potentially made to central fabrication facilities to help improve socket quality.

\section{CONCLUSIONS}

A very precise assessment was conducted of the match between sockets made by central fabrication facilities and the corresponding electronic AAOP files. Results from 10 companies showed a considerable variability in quality. Some companies consistently made good sockets, some companies consistently made poor sockets, and some companies sometimes made good sockets. For some companies, the errors were consistent. Accommodating consistent oversizing or undersizing is relatively easy in clinical practice. However, inconsistent sizing or shape distortion, as occurred for some of the companies, is more difficult to tolerate and the most challenging for central fabrication facilities.

\section{ACKNOWLEDGMENTS}

Assistance from Brian Hafner, $\mathrm{PhD}$, in image preparation is appreciated.

This material was based on work supported by the National Institutes of Health (NIH), National Institute of Bioimaging and Biomedical Engineering, grant NIH EB07329.

The authors have declared that no competing interests exist.

\section{REFERENCES}

1. Childress DS. Presentation highlights: Computer-Aided Design and Manufacture (CAD-CAM). J Rehabil Res Dev. 2002;39(3 Suppl):15-16.

2. Smith DG, Burgess EM. The use of CAD/CAM technology in prosthetics and orthotics-Current clinical models and a view to the future. J Rehabil Res Dev. 2001;38(3): 327-34. [PMID: 11440264]

3. Sanders JE, Mitchell SB, Zachariah SG, Wu K. A digitizer with exceptional accuracy for use in prosthetics research: A technical note. J Rehabil Res Dev. 2003;40(2):191-95.

[PMID: 15077643]

4. Zachariah SG, Sorenson E, Sanders JE. A method for aligning trans-tibial residual limb shapes so as to identify regions of shape change. IEEE Trans Neural Syst Rehabil Eng. 2005;13(4):551-57. [PMID: 16425837]

5. Veltkamp RC, Hagedoorn M. State-of-the-art in shape matching. Utrecht (the Netherlands): Utrecht University; 1999.

6. Fernie GR, Holliday PJ. Volume fluctuations in the residual limbs of lower limb amputees. Arch Phys Med Rehabil. 1982;63(4):162-65. [PMID: 7082139]

Submitted for publication June 15, 2006. Accepted in revised form February 12, 2007. 